\title{
Social Network Mental Disorders Detection via Online Social Media Mining
}

\author{
Prof. Narinder Kaur ${ }^{1} \mid$ Lakshay Monga ${ }^{2}$
}

\begin{abstract}
${ }^{1}$ Assistant Professor, Department of Information Technology, Maharaja Agrasen Institute of Technology, Delhi, India.
\end{abstract} ${ }^{2}$ Student, Department of Information Technology, Maharaja Agrasen Institute of Technology, Delhi, India.

\section{To Cite this Article}

Prof. Narinder Kaur and Lakshay Monga, "Social Network Mental Disorders Detection via Online Social Media Mining", International Journal for Modern Trends in Science and Technology, Vol. 07, Issue 01, January 2021, pp.-27-30.

\section{Article Info}

Received on 22-November-2020, Revised on 18-December-2020, Accepted on 22-December-2020, Published on 28-December-2020.

\section{ABSTRACT}

Social Network Mental Disorder Detection" or "SNMD" is an approach to analyse data and retrieve sentiment that it embodies. Twitter SNMD analysis is an application of sentiment analysis on data from Twitter (tweets), in order to extract sentiments conveyed by the user. In this paper, we aim to review some papers regarding research in sentiment analysis on Twitter, describing the methodologies adopted and models applied, along with describing a generalized Python based approach. A prototype system is developed and tested.

KEYWORDS: Social Media Mining, Sentiment Analysis, Machine Learning.

\section{INTRODUCTION}

Social Network Mental Disorders Detection Analysis is a technique widely used in text mining. Social Network Mental Disorders Detection Analysis, therefore means, using advanced text mining techniques to analyze the sentiment of the text (here, tweet) in the form of positive, negative and neutral. It is also known as Opinion Mining, is primarily for analyzing conversations, opinions, and sharing of views (all in the form of tweets) for deciding business strategy, political analysis, and also for assessing public actions.

Social Network Mental Disorders Detection analysis is a process of deriving sentiment of a particular statement or sentence. It's a classification technique which derives opinion from the tweets and formulates a sentiment and on the basis of which, sentiment classification is performed. Sentiments are subjective to the topic of interest. We are required to formulate that what kind of features will decide for the sentiment it embodies. In the programming model, sentiment we refer to, is class of entities that the person performing sentiment analysis wants to find in the tweets. The dimension of the sentiment class is crucial factor in deciding the efficiency of the model. For example, we can have two-class tweet sentiment classification (positive and negative) or three class tweet sentiment classification (positive, negative and neutral). Sentiment analysis approaches can be broadly categorized in two classes - lexicon based and machine learning based. Lexicon based approach is unsupervised as it proposes to perform analysis using lexicons and a scoring method to evaluate opinions. Whereas machine learning approach involves use of feature extraction. 


\section{THEORY}

The passive-aggressive algorithms are a family of algorithms for large-scale learning. They are similar to the Perceptron in that they do not require a learning rate. However, contrary to the Perceptron, they include a regularization parameter $\mathrm{C}$.

For classification, PassiveAggressiveClassifier can be used with loss='hinge (PA-I) or loss = 'square_hinge' (PA-II).

For regression, PassiveAgressiveRegressor can be used with loss='epsilon_insensitive' (PA-I) or loss = 'squared_epsilon_insensitive' (PA-II).

\section{The Passive-Aggressive Algorithm}

- Each example defines a set of consistent hypotheses: $\quad C_{\varepsilon}\left(\mathrm{z}_{t}\right)=\left\{\mathbf{w} \mid \delta\left(\mathbf{w} ; \mathbf{z}_{t}\right) \leq \varepsilon\right\}$

- The new vector $\mathbf{w}_{t+1}$ is set to be the projection of $\mathbf{w}_{t}$ onto $C_{\varepsilon}\left(\mathbf{z}_{t}\right)$

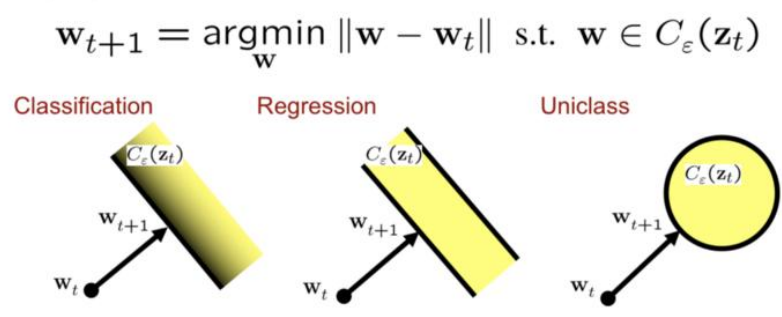

Figure 1. Passive-Aggressive Algorithm

Let me start with the case of classification. On each round we receive an instance $\mathrm{x} t$ and extend a prediction using our current hypothesis $\mathrm{w} t$. We then receive the true target yt and suffer an instantaneous loss based on the discrepancy between yt and our prediction. Our goal is to make the cumulative loss that we suffer small. Finally, we update the hypothesis according to the previous hypothesis and the current example. The setting for regression and uniclass is similar. In Regression, we predict a real value target $\mathrm{w} t{ }^{*} \mathrm{x} t$ and then suffer loss according to the discrepancy between our prediction and the true one. In Uniclass, the setting is slightly different as we do not have instances. Instead, we extend a center point $\mathrm{w} t$ and hope that $\mathrm{y} t$ falls within a radius of epsilon from $w t$. We again suffer loss according to the actual distance between $y t$ and the center $\mathrm{w} t$. The three problems described above can be viewed as a private case of the following general framework. The end result is a common algorithmic framework and an accompanying analysis. We denote the discrepancy of a hypothesis $\mathrm{w}$ according to example $z t$ by delta. For classification, delta is the negation of the signed margin, and for regression and classification delta is the distance between our prediction and the true target. We start with algorithm and analysis for the realizable case - that is we assume that there exist w star and epsilon star such that We define the hinge-loss to be the maximum between delta minus epsilon and zero. Say in other words, we pay linearly on the violation of the constraint: delta less or equal to epsilon. unified view:-

- Define discrepancy for $\mathbf{z}_{t}$ :

$$
\delta\left(\mathbf{w} ; \mathbf{z}_{t}\right)=\left\{\begin{array}{c}
-y_{t} \mathbf{w} \cdot \mathbf{x}_{t} \\
\left|y_{t}-\mathbf{w}_{t} \cdot \mathbf{x}_{t}\right| \\
\left\|\mathbf{y}_{t}-\mathbf{w}_{t}\right\|
\end{array}\right.
$$

- Unified Hinge-Loss:

$$
\ell_{\varepsilon}\left(\mathbf{w} ; \mathbf{z}_{t}\right)=\left[\delta\left(\mathbf{w} ; \mathbf{z}_{t}\right)-\varepsilon\right]_{+}=\max \left\{0, \delta\left(\mathbf{w} ; \mathbf{z}_{t}\right)-\varepsilon\right\}
$$

- Notion of Realizability: $\forall_{t}, \quad \delta\left(\mathbf{w}^{*} ; \mathbf{z}_{t}\right) \leq \varepsilon^{*}$

\section{Figure 2. Unified View}

\section{LITERATURE SURVEY}

In this section we will go through the history of mental health analysis in social media in multiple aspects.

\section{Multimodal mental health analysis in social media}

Depression is a major public health concern in the U.S. and globally. While successful early identification and treatment can lead to many positive health and behavioral outcomes, depression, remains undiagnosed, untreated or undertreated due to several reasons, including denial of the illness as well as cultural and social stigma. With the ubiquity of social media platforms, millions of people are now sharing their online persona by expressing their thoughts, moods, emotions, and even their daily struggles with mental health on social media. Unlike traditional observational cohort studies conducted through questionnaires and self-reported surveys, we explore the reliable detection of depressive symptoms from tweets obtained, unobtrusively. Particularly, we examine and exploit multimodal big (social) data to discern depressive behaviors using a wide variety of features including individual-level demographics. By developing a multimodal framework and employing statistical techniques to fuse heterogeneous sets of features obtained through the processing of visual, textual, and user interaction data, we significantly enhance 
the current state-of-the-art approaches for identifying depressed individuals on Twitter (improving the average F1-Score by 5 percent) as well as facilitate demographic inferences from social media. Besides providing insights into the relationship between demographics and mental health, our research assists in the design of a new breed of demographic-aware health interventions.

\section{Qualitative language analysis}

The recent LIWC version [86] summarizes textual content in terms of language variables such as analytical thinking, clout, authenticity, and emotional tone. It also measures other linguistic dimensions such as descriptor categories (e.g., percent of target words gleaned from the dictionary, or words longer than six letters-Sixltr), informal language markers (e.g., swear words, netspeak), and other linguistic aspects (e.g., first person singular pronouns). Thinking Style: The words we use to communicate can reveal our style of thinking. There are two common approaches for extracting an individual's thinking style. First, measuring one's natural way of trying to understand, analyze, and organize complex events has a strong association with analytical, formal, and logical thinking. LIWC relates higher analytic thinking to more formal and logical reasoning, whereas a lower value indicates a focus on narratives. Second, cognitive processing, which measures problem solving in the mind, is captured through words such as "think," "believe," "realize," and "know" and demonstrates "certainty" in communication. High values for analytical thinking implies clarity of thought.

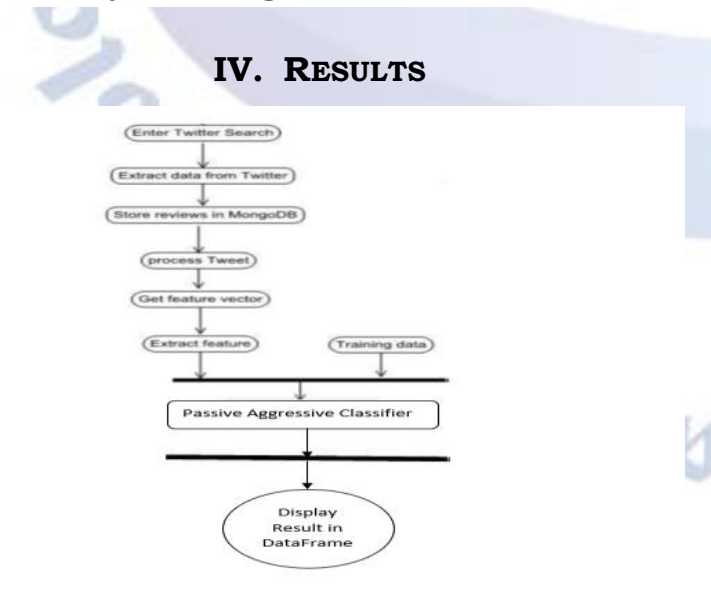

Figure 3. Flow Chart of the Proposed System

We built our system and used Passive Aggressive Classifier model in it. It can detect sentiment of the text in real-time. We haved used Twitter API to get Live tweets, it can help to detect where is action mandatory to detect if a person is in depressed state or not.

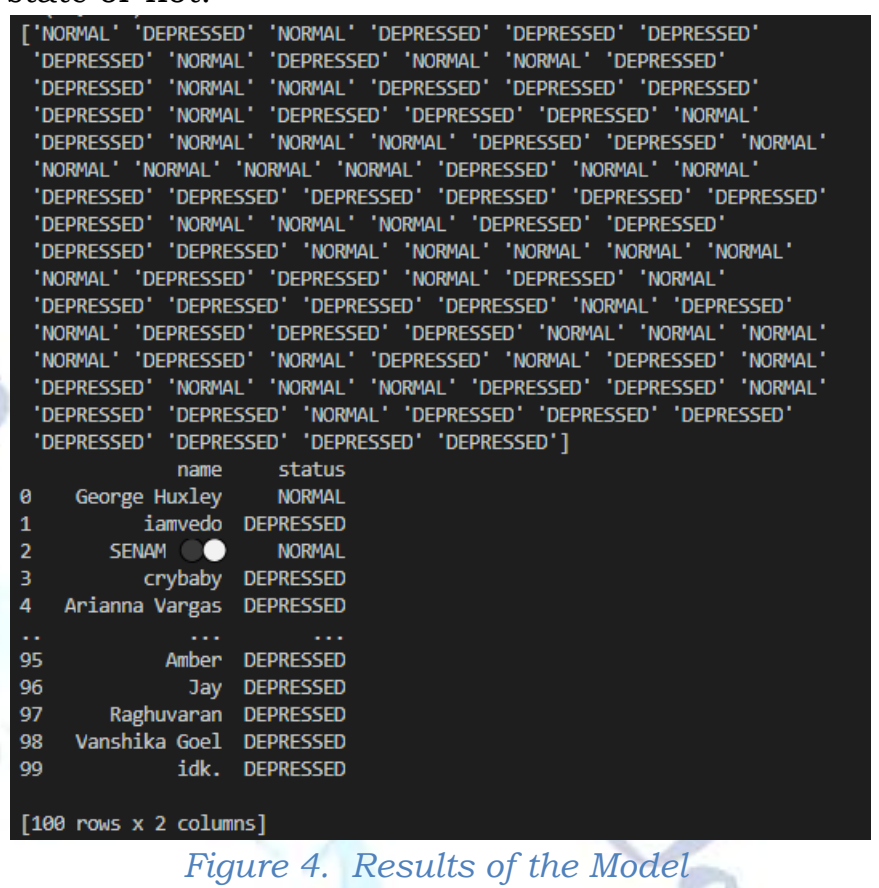

\section{Conclusions}

Social Network Mental Disorders Detection Analysis is an interesting way to think about the applicability of Natural Language Processing in making automated conclusions about text. It is being utilized in social media trend analysis and, sometimes, for marketing purposes. Making a SNMD Analysis program in Python is not a difficult task, thanks to modern-day, ready-for-use libraries. This program is a simple explanation to how this kind of application works. This is only for academic purposes, as the program described here is by no means production- level. This research topic has evolved during the last decade with models reaching the efficiency of almost $85 \%-90 \%$. But it still lacks the dimension of diversity in the data. Along with this it has a lot of application issues with the slang used and the short forms of words. Many analysers don't perform well when the number of classes are increased. Also it's still not tested that how accurate the model will be for topics other than the one in consideration.

\section{FUTURE WORK}

Use of parser can be embedded into system to improve results. A web based application can be made for our work in future. We can improve our system that can deal with sentences of multiple meanings. We can also increase the classification categories so that we can get better results. We can start work on multi-languages like Hindi, Spanish, 
etc. to provide sentiment analysis to more local languages.

\section{REFERENCES}

[1] Multimodal mental health analysis in social media By Amir Hossein Yazdavar, Mohammad Saeid Mahdavinejad, Goonmeet Bajaj, William Romine, Amit Sheth, Amir Hassan Monadjemi, Krishnaprasad Thirunarayan, John M. Meddar, Annie Myers, Jyotishman Pathak, Pascal Hitzler.

[2] Deep learning in mental health outcome research: a scoping review By - Chang Su, Zhenxing Xu, Jyotishman Pathak \& Fei Wang.

[3] Social Network Mental Disorder Detection Based Markov Model By - Teena Patidar1, Prof. Avinash Sharma2 1PG Scholar, 2Assistant Professor Department of Computer Science Engineering, MITS, Bhopal, Madhya Pradesh, India. 